\title{
Arteriovenous Relationships in the Pathogenesis of Encephalopathy
}

\author{
Alexander G. Kruglov*, PhD, ScD; Alexander Yu. Vasiliev, PhD, ScD; \\ Veronica A. Sherman \\ Moscow State University of Medicine and Dentistry \\ Moscow, Russia
}

\begin{abstract}
The study aims at investigating the disturbance in intra- and extracranial interaction of arterial and venous vessels in stable arterial hypertension (SAH) and building a model of vascular relationships in the system: inflow - the exchange field of cerebral blood flow - outflow. Baseline data were obtained by catheterization through a probe that was wedged in the superior bulb of the internal jugular vein, where the hemodynamic and biochemical parameters of cerebral blood flow were obtained. Arterial blood was collected from the thoracic aorta. We performed the correlation and factor analyses of the relationship between the parameters of inflow and outflow to the skull in SAH patients compared with those in the control group. The identified differences led to the following conclusions: There is a loss of homeostatic control for the hemodynamic (extra- and intracerebral) and biochemical regulation in SAH; the high-energy processes of the aortic chamber (systolic and pulse pressure) spread to the bloodstream of the brain; the damping function of carotid siphons is impaired; cerebral venous stasis is formed; increased pressure in the microvascular venous network of the brain is defined; and a loss of the homeostatic control of the rheological properties of blood is defined. The loss of extracranial regulation of intracranial venous pressure in SAH leads to venous plethora of the intracerebral vessels, increasing the "booster" pressure in the microvasculature, and circulatory hypoxia of brain tissues. The consequences of these changes are metabolic and hemodynamic disturbances in energy supply for activated neurons, as well as circulatory hypoxia resulting in disturbances of the regulatory function of the nervous system and mental activity, and the development of hypertonic angioencephalopathy. (Int J Biomed. 2015;5(3):127-131.)
\end{abstract}

Keywords: cerebral venous stasis; circulatory hypoxia; homeostatic control; hypertonic angioencephalopathy.

Abbreviations: Ao, aorta; BP, blood pressure; CO, cardiac output; CBF, cerebral blood flow; CASs, carotid artery siphons; DP, diastolic pressure; EDP, end-diastolic pressure; HAE, hypertonic angioencephalopathy; IJV, internal jugular vein; IVP, intracranial venous pressure; $\mathbf{L V}$, left ventricle; $\mathbf{M}$, mean value; $\mathbf{m}$, confidence interval ratio; MP, mean pressure; PP, pulse pressure; RV, right ventricle; RA, right atrium; SS, sigmoid sinus; SP, systolic pressure; SAH, stable arterial hypertension; SV, stroke volume.

\section{Introduction}

The study of the relationships of the intracranial venous pressure with systemic arterial pressure and central aortic pressure in stable arterial hypertension (SAH), compared to the norm, is a main issue of this work. We built a model of the impact of the intracranial and cerebral blood flow on the development of disturbances in neuron activity and hypertonic angioencephalopathy (HAE) formation. Despite the importance of the problem, there are a small number of studies dedicated to impairments in the cerebral venous

*Corresponding author: Alexander G. Kruglov, PhD, ScD. Moscow State University of Medicine and Dentistry, Moscow, Russia E-mail:krag48@mail.ru system in HAE. There is no consensus about the primary or secondary nature of venous pathology in arterial hypertension. Modern concepts of HAE pathogenesis mainly associate the clinical manifestations with the active processes in the arterial vascular bed. In particular, ischemic attack, "small" strokes, and progressive cerebral ischemia are considered as the main causes of HAE. There is a great deal of literature dedicated to changes in the structure and functions of intra- and extracerebral arteries and only a few studies dedicated to the role of the cerebral venous vascular bed in the development of HAE. In one such study, B. Mashsin et al have found a brain venous hemodynamic abnormality with an increase in blood flow volume and decrease in venous velocity, as well as an increase of the linear velocity in the arterial flow [1].

The purpose of this study was to create a model of 
relationships between the intracranial/cerebral arterial and venous flows both among themselves and with the systemic systolic pressure and central aortic pressure, and create a central concept of HAE pathogenesis, taking into account the abnormalities of the metabolic support for the actualized neural networks and related changes in the mental sphere.

\section{Methods and Results}

For all participants, the study was conducted in the same laboratory by the same methods. The data were obtained from patients in the supine position by catheterization through a probe that was wedged in the upper bulb of the internal jugular vein (IJV). The results indicate the levels of blood pressure (BP) in the venous system of the brain and sigmoid sinus (SS) (Tables 1 and 2).

Table 1.

Pressure levels (mmHg) in SS (IVP)

\begin{tabular}{|c|c|c|c|c|c|}
\hline & & SP & DP & PP & MP \\
\hline \multirow{4}{*}{ Norm } & $\mathrm{M}$ & 10.5 & 8.3 & 2.4 & 9.4 \\
\cline { 2 - 6 } & $\mathrm{m}$ & 2.2 & 1.8 & 0.75 & 2.2 \\
\cline { 2 - 6 } & $\mathrm{Max}$ & 21 & 16.8 & 4.2 & 18.9 \\
\cline { 2 - 6 } & $\mathrm{Min}$ & 6 & 3.9 & 0.5 & 4.9 \\
\hline \multirow{4}{*}{ HAE } & $\mathrm{M}$ & 11.5 & 7.7 & 3.8 & 10.1 \\
\cline { 2 - 6 } & $\mathrm{m}$ & 3.2 & 2.9 & 1.4 & 3.9 \\
\cline { 2 - 6 } & Max & 22.6 & 19.2 & 7 & 20.4 \\
\cline { 2 - 6 } & Min & 3.9 & 2.1 & 1.3 & 2.4 \\
\hline
\end{tabular}

Table 2.

Pressure levels $(\mathrm{mmHg})$ in IJV

\begin{tabular}{|c|c|c|c|c|c|}
\hline & & SP & DP & PP & MP \\
\hline \multirow{4}{*}{ Norm } & M & 7.3 & 5.5 & 2.1 & 6.7 \\
\cline { 2 - 6 } & $\mathrm{m}$ & 1.1 & 1 & 0.6 & 1.3 \\
\cline { 2 - 6 } & Max & 13.8 & 10.5 & 5.1 & 11.7 \\
\cline { 2 - 6 } & Min & 2.4 & 2 & 0.2 & 1.4 \\
\hline \multirow{4}{*}{ HAE } & $\mathrm{M}$ & 8.7 & 6.3 & 2.6 & 7.2 \\
\cline { 2 - 6 } & $\mathrm{m}$ & 3.4 & 3.3 & 1.1 & 3.2 \\
\cline { 2 - 6 } & Max & 21 & 14. & 6.4 & 15.7 \\
\cline { 2 - 6 } & Min & 2.4 & 0.5 & 1.2 & 2.1 \\
\hline
\end{tabular}

Catheterization was performed by percutaneous puncture of the right femoral artery and the right femoral vein using the Seldinger technique. BP was recorded in the thoracic aorta. The study was conducted under X-ray control. We evaluated the results obtained in patients with essential hypertension grade 2 and resistant to therapy. Since preliminary analysis showed no significant differences among patients, we considered the study group as a homogeneous group with SAH. The study included 61 patients. To compare the results, data of 60 people in the control group were obtained and processed by methods of mathematical statistics in a similar way. The detailed description of the groups and methods of the results processing were shown previously [2]. For intracranial venous pressure (IVP) in SAH, we note despite being within the boundaries of the "zone of norm," a reliable growth of pressure level (by $8 \%$ to $9 \%$ ), especially significant for the pulse pressure (by 58\%). Presence of the pressure gradient between the intra- and extracranial venous systems, despite the varying levels of pressure in the IJV (the effect of intrathoracic and barometric pressure), proves that IVP is formed with the participation of the factors affecting in IJV. Previously [6], we have observed a radical change in the composition and quality of the correlations for intra- and extracranial blood flow with other parameters in SAH. Particularly important are the loss or inversion of correlations with the parameters of general and plasma viscosity and rheology, as well as the loss of correlations with the final gas exchange products of the brain that determine the plasma gas tension $\left(\mathrm{O}_{2}, \mathrm{CO}_{2}\right)$.

All levels of pressure in the right atrium (RA) in SAH (out MP) significantly exceed the boundaries of the normal zone (Table 3). Pressure levels in Ao and LV are shown in Table 4.

Table 3.

Pressure levels ( $\mathrm{mmHg}$ ) in $\mathrm{RA}$

\begin{tabular}{|c|c|c|c|c|c|c|c|c|}
\hline & & \multicolumn{5}{|c|}{ RA } & \multicolumn{2}{|c|}{ LV } \\
\hline & & A & $X$ & V & Y & MP & EDP & MP \\
\hline \multirow{4}{*}{ Norm } & M & 5.7 & 2.4 & 4.6 & 3.4 & 4.1 & 5.1 & 14.4 \\
\hline & $\mathrm{m}$ & 0.9 & 0.7 & 0.8 & 0.2 & 1.1 & 1.2 & 1.8 \\
\hline & Max & 8.8 & 5.3 & 6.5 & 6.0 & 8.4 & 10 & 19.8 \\
\hline & Min & 3.0 & 0 & 1.6 & 0 & 1.2 & 0 & 8.8 \\
\hline \multirow{4}{*}{ HAE } & $\mathrm{M}$ & 7.4 & 4.0 & 6.4 & 4.7 & 3.4 & 3.4 & 13.9 \\
\hline & $\mathrm{m}$ & 2.7 & 2.1 & 2.8 & 2.0 & 2.6 & 2.6 & 4.4 \\
\hline & Max & 11.8 & 9.0 & 14.1 & 9.0 & 8.4 & 8.4 & 28 \\
\hline & Min & 1.8 & 0 & 1.8 & 0 & 0 & 0 & 6.6 \\
\hline
\end{tabular}

Table 4.

Pressure levels (mmHg) in Ao and $\mathrm{LV}$

\begin{tabular}{|c|c|c|c|c|c|c|c|c|c|}
\hline & & \multirow{2}{*}{$\mathrm{CO}$} & \multirow{2}{*}{ SV } & \multicolumn{4}{|c|}{ Ao } & \multicolumn{2}{|c|}{ LV } \\
\hline & & & & $\mathrm{SP}$ & DP & PP & MP & SP & PP \\
\hline \multirow{4}{*}{ Norm } & $\mathrm{M}$ & 10.9 & 128.4 & 114 & 71 & 40.3 & 92.6 & 108 & 100.5 \\
\hline & $\mathrm{m}$ & 1.3 & 22.4 & 4.5 & 3.8 & 3.5 & 5.1 & 6.4 & 7.1 \\
\hline & Max & 18.7 & 186 & 147 & 87 & 65 & 115 & 130 & 130 \\
\hline & Min & 5.5 & 80 & 90 & 50 & 21 & 70 & 90 & 85 \\
\hline \multirow{4}{*}{ HAE } & M & 11.1 & 147 & 180 & 101 & 79 & 137 & 180 & 168 \\
\hline & $\mathrm{m}$ & 3.1 & 38,9 & 31 & 21 & 22 & 23 & 34 & 28 \\
\hline & Max & 21.7 & 246 & 270 & 155 & 144 & 210 & 260 & 248 \\
\hline & Min & 5.3 & 50 & 150 & 78 & 40 & 96 & 150 & 138 \\
\hline
\end{tabular}

Previously, we analyzed correlations between parameters of RA pressure and indicators of IVP, as well as 
between the biochemical parameters (including rheological parameters) of blood flowing from the brain [6]. We found a loss of correlations for pressure among the parameters of general and plasma viscosity, vasoactive regulators of cerebral blood flow (electrolytes), and final metabolites of the brain (arteriovenous difference in $\mathrm{O}_{2}$ and $\mathrm{HbO}_{2}$ ) in SAH. In other words, during development of SAH there is a loss of regulatory relationships of the RA and IVP with brain metabolites and rheological parameters in a single hemodynamical chamber (IVP--RA), where the main regulator, in our opinion, is the minimal pressure in the outflow tract from the brain: in the RA. These data are well known and shown for complete characterization of the study issue. In norm, the active process of the left ventricle (LV) systole (systolic and pulse pressure in aorta (Ao) and LV) have no correlations with the pulse pressure (PP) in SS (Table 5). LV systolic and pulse pressures are in negative association with systolic, diastolic and mean pressure of SS. Consequently, the formation of IVP in norm does not depend on Ao PP, at the same time it has a relationship with the systolic, diastolic and mean pressure in Ao. In SAH, there is a loss of the correlation between IVP and the pressure in the aortic chamber, but a correlation between the active phase of LV and SS PP is found. Thus, in norm, IVP levels correlate with the LV ejection phase; maximum of pressure in system VS--Ao is achieved with opening of the aortic valve; maximal pressure for the skull (brain) is formed; a wave process in Ao (immediate application area is extra- and intracranial blood vessels) is generated.

\section{Table 5 .}

Correlations between IVP and Ao - LV

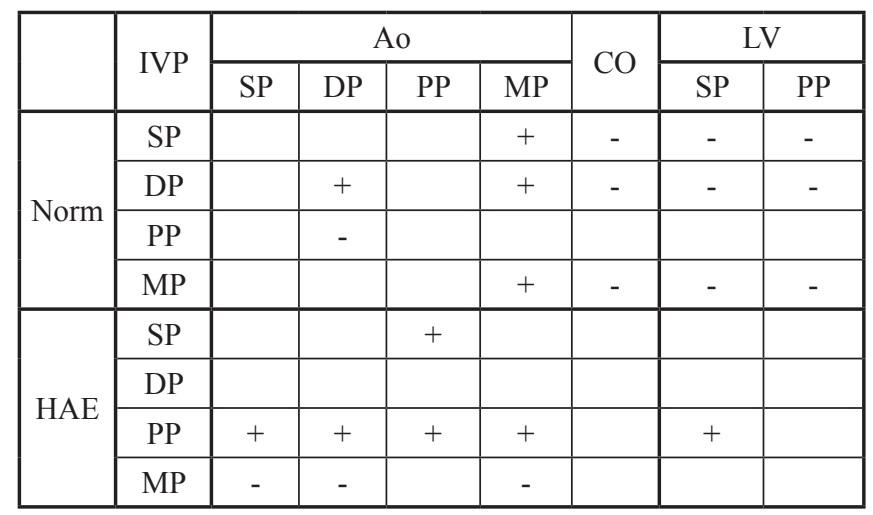

In the horizontal position, LV systole, forming a rotational and translational movement around the outer (variable) axis in the form of a spherical wave structure [3] of aortic flow, reaches all areas of the body before closing the aortic valve [4]. The negative correlation with the levels of IVP is explained only by the existence of a characteristic structure between the cavity of the skull and extracranial vessels, which changes its clearance under the influence of the wave process. We believe that this structure is carotid artery siphons (CASs) located in the cavernous sinus. CASs are guides for wave structures of the heart; they are informative characteristics of different levels of regulation [2] interfering in the cavernous sinuses of the dura mater.
The presence of CASs explains the negative relations between the IVP and LV ejection phase in norm. This relation changes diametrically in SAH and becomes positive, which indicates parallel changes in IVP and Ao pressure, which are possible only as a result of changes of functional relationships in the system CASs--cavernous sinus which is connected through the sinus petrosi inferior with the zone of catheter wedging while obtaining the initial data. It should be also noted that the sympathetic plexus of CASs participates in innervations of the eyeball, which plays an important role in purposeful behavior. The increased IVP in SAH is necessary to maintain the pressure gradient at the required level to ensure the adequate perfusion and cerebral metabolic processes (in the zone of norm). This is possible only if the ability to the extracranial regulation (the defeated CASs) is lost, as well as the lesion of the intracranial vascular bed with narrowing of the lumen and rigidity of vascular wall.

We consider the phase of aortic peak during Ao and LV separation as 1) a final stage of the high-energy phase and 2) an initial stage of the diastolic evolution. The action of CASs, whose contraction is caused by LV systole, makes a "selection" (damping) of the pressure, starting with a value equal to the average BP. Pressure levels exceeding the average BP in norm remain in "reserve" to respond in stressful situations (in principle, it is quite a minimal adaptive response, namely, the diminution of CAS reaction to the aortic wave process). First of all, the average BP is positively associated with ICP up to diastolic BP, which indicates the setting of the unified hydrodynamic system (Ao - vessels entering the skull - IVP) in the phase of the diastolic evolution (from average to diastolic pressure). This explains the positive relationship of the diastolic evolution of the intracranial venous bed with the aortic diastolic evolution. Achieving minimal values by the Ao-IVP gradient is replaced by the LV systole. In other words, Ao pressure--IVP relationships change their own correlation from the negative through "zero" to the positive during one cardiac cycle. The radical difference for SAH is: 1) the dependence of IVP only on the high-energy phase with an inversion of direction of regulation; 2) the lack of correlation at all stages of the diastolic evolution; 3) the lack of a reserve of pressure for urgent adaptation. Thus, during SAH development, despite the increase in energy expenditure to ensure the cerebral blood flow and metabolism, the efficiency of metabolic support for the brain functioning is reduced. Our data [2] suggest that during SAH development, there is a loss of the hemodynamic mechanisms for correction of the negative manifestations of hyperfibrinogenemia, rheological and viscous blood parameters, and thrombus formation; ie, there is a loss of regulation of conjugation between thrombotic factors and pressure towards the outflow from the brain, both of which exist in norm. Pathways of the venous outflow according to the pressure gradient from the cortex through the venous sinuses in the RA have no valves, making a unified hemodynamic chamber [2]. The pressure at the outlet of the intracerebral venous bed in the zone of minimal pressure significantly increases the entire range of values in SAH. The critical value for intravascular pressure in veins depends on the ratio of wall thickness to the radius of the vessel and elastic 
modulus. Loss of stability and a sharp change in the lumen of the intracranial veins occurs at pressure changes by 0.2 to 0.5 $\mathrm{Hg}$, resulting in a change in the throughput volume capability and the cross-sectional shapes of venules and veins. A gradient of transmural pressure effects in the system "liquor--veins" is possible in both directions, and an increase in venous pressure, causing the maximal volume flow, increases the transmission pressure on the liquor. The decreased venous blood flow, which is accompanied by liquor hypertension, is found in SAH. The increase in venous stasis is a marker of decompensation of cerebral circulation. A disparity between the rate of arterial inflow and venous return (delay) is more pronounced in patients with chronic heart failure [5]. We believe that in a unified hydrodynamic chamber with the outflow according to the pressure gradient, the disturbances in any portion of unified hemodynamic space "intracerebral veins--RA" [2] affect the condition and regulation of the entire intra- and extracerebral venous flow regardless of the exposure vector (along or against the blood flow). Increasing the "booster" venous pressure in the valveless bloodstream inevitably leads to increased pressure in the main outflow tracts from the brain ((1) superior sagittal sinus - bridging veins - cortical cuff cortical veins, (2) straight sinus - deep cerebral veins -cortical veins) against the blood flow to the functional unit of the microvasculature network: post-capillary venule - capillary pre-capillary arterioles. The increase in the "booster" venous pressure according to the previously described mechanism [6] is spread through valveless veins on the entire volume of the brain, and affects the liquor pressure in accordance with the transfer mechanism.

The minimum dimensions of the neural structures, in which there are the marked changes in blood flow (functional hyperemia--FH) under functional load, correspond to the size of neural columns. According to G. Mchedlishvili [7], the initial stage of $\mathrm{FH}$ has neurogenic regulation, but the $\mathrm{K} / \mathrm{Na}$ pump having the mechanisms of metabolic supply for neurons has almost the same time parameters. During development of the activity in the exchange field of the actualized neural network (the excited neurons), the venous vessels eliminating metabolites are already in a state of maximum volumetric capacity without the possibility to remove the additional blood volume by changing the volume of the venous vessels.

The decreased rate of blood flow in the microvasculature cannot be compensated for by a Fareusa-Lindqvist effect. Additional complicating factors of blood flow in the macroand microcirculation networks are disorders in aggregation, rheology and viscosity properties of blood with the loss of homeostatic control, and loss of regulation of the hemodynamic and biochemical interactions, which take place in norm [2]. All of this results in a delay in metabolite elimination, $\mathrm{O}_{2}$ deficiency, an excess of $\mathrm{CO}_{2}$, a lack of glucose, changes in intra- and extravascular concentration of electrolytes (regardless of the activity of transmembrane channels), increased platelet activity, and other abnormal reactions (ie, all of the above are indicators of the development of local circulatory hypoxia). Disturbances in the metabolism of neurons, which occur due to the lack of substrates for oxidation, lead to a reduced formation of macroergic substances (ATP,
ADP, creatine phosphate). Accumulation of metabolites is a disturbing factor. Thus, with an increase in the activity of neurons and formation of activated neural associations, there is a local circulatory hypoxia. Manifestations of circulatory hypoxia are the changes in activity of selective channels of membranes, and in their permeability (especially for $\mathrm{Na}, \mathrm{K}$, and $\mathrm{Ca}$ ) and excitability, and destruction of structural patterns of neurons (nucleus, organelles, and cytoplasm). The excess of the oxidized metabolites plays a special role in damaging cellular and intracellular membranes. A result of this excess is characterized by the development and progression of degenerative processes in neurons, impaired intracellular signaling (both in neurons and the adjustable effector cells), a change of phosphorylation and the activity of functional proteins. Many researchers characterize the disturbances in the energy supply of $\mathrm{K} / \mathrm{Na}, \mathrm{Ca}$ membrane pumps leading to abnormalities in the transmembrane gradient of electrolyte concentrations as one of the main mechanisms of neuronal hypoxia. These disturbances lead to membrane depolarization and inactivation of the transmembrane channels. Abnormalities in the calcium pump reduce $\mathrm{Ca}$ release from cytosol to the extracellular environment with deposition in endoplasmic reticulum. There is a reduction of FH in the projection area of the conditioned response caused by a decrease in the blood supply to the cortex in SAH [8]. The total result of the above-described is a disturbance of the integrative activity of the nervous system in different forms: the pathological determinants, the pathological dominants, the pathological systems and others [9], as well as the suppression of neuronal population activity [10].

In hypoxic changes of cortex, hyperexcitability (a decreased threshold) and metabolic acidosis, the sensory stimulus for activation and the subsequent activity of neural networks have a lower (compared with the norm) power and duration of the signal for initiating the operation of the "answer" mechanism and metabolic supply for the exchange fields of the involved neurons. The reduced energy level of the initial signal means an increase in the total amount of information and the range of the readable signals from the environment.

The increase in size of the information field and excessive sensory stimulation (as a consequence) associated with difficulty in selecting the main control signal leads to excessive stimulation of the synapses of the afferent impulses and changes in the efficiency of synaptic transmission in the form of long-term potentiation and depression (pathological excitation or inhibition). Abnormalities in the allocation of the main control signals lead to the deficit model of reality distortion and the disturbance of translational symmetry. Formation of an objective reality model having an information deficit and mismatch leads to discrepancies in the projection mechanisms to the real parameters of the environment, as well as disturbances of the adequacy and determinism in the system "environment - man - environment." Projection is a way of interacting, adapting, and searching for methods to maintain homeostatic equilibrium ("Deterministic equivalent response" according to Le Chatelier). Having a structural mismatch to the original information field (objective reality) 
the "projection" (cognitive product) is not equivalent, but rather asymmetric, to the content of the external world, with a growing parametric discrepancy. The consequence of this is: an increasing mismatch in stimulus-response; stereotyping of behavioral forms (the result of the stable existence of neural associations of standard answer); a decrease in the adaptive arsenal; and adjustment disorder (physiological, psychological, and social).

\section{Conclusion}

There is a loss of homeostatic control for the hemodynamic (extra- and intracerebral) and biochemical regulation in $\mathrm{SAH}$; the damping function of carotid siphons is impaired; the high-energy processes of the aortic chamber (systolic and pulse pressure) spread to the bloodstream of the brain; cerebral venous stasis is formed; increased pressure in the microvascular network of the brain is defined; and a loss of the homeostatic control of the rheological properties of blood is defined. The loss of extracranial regulation of intracranial venous pressure in SAH leads to venous plethora of the intracerebral vessels, increasing the "booster" pressure in the microvasculature, and circulatory hypoxia of brain tissues. The consequences of these changes are metabolic and hemodynamic disturbances in energy supply for activated neurons, as well as circulatory hypoxia resulting in disturbances of the regulatory function of the nervous system and mental activity, and the development of HAE.

\section{Competing interests}

The authors declare that they have no competing interests.

\section{References}

1. Machin VV, Belova LA, Belov VG, Salikhova EA. Cerebral discirculation in arterial hypertension (clinical and ultrasound). Klin Fiziol Krov. 2009;1:40-4. [Article in Russian]. 2. Kruglov A,Gebel G, Vasilyev A. Intracranial venous pressure as possible determinant of mental regulation. Int J Biomed. 2011;1(4):245-8. [Article in Russian].

3. Kuzmina NB, Drogaicev AD. Formation of blood flow in the cavity of the left ventricle. In book: Cardiac physiology. Series: Manual of physiology; 1980:212-4. [in Russian].

4. Lightfoot A. Transport phenomena in live systems. Biomedical aspects of momentum and mass transport. M.: Mir, 1977. [in Russian],

5. Zolotukhina NS. Hypertensive encephalopathy: venous brain discirculation in patients with chronic heart failure. Abstract of PhD Thesis. Moscow; 2006. [in Russian]

6. Kruglov A, Gebel G, Vasilyev A. Impact of intra extracranial hemodynamics on cerebral ischemia by arterial hypertension (Part 1-2). Int J Biomed. 2012;2(2):89-101. [Article in Russian].

7. Mchedlishvili GI. Microcirculation. M.: Nauka; 1989. [in Russian].

8. Khananashvili YaA. Blood supply of the cerebral cortex and its bioelectric activity in the dynamics of arterial hypertension. Abstract of ScD Thesis. Leningrad; 1990. [in Russian]

9. Kryzhanovsky GN. Discirculatory pathology of the nervous system. Moscow: MedInform Agentstvo; 2009. [in Russian].

10. Levin SG, Shamsudinov AA, Goduhin OV. Comparative effect of blockers of ATP-sensitive $\mathrm{K}+$-channels on the induced changes in activity of pyramidal neurons of hippocampal area CA1 under brief episodes of hypoxia. Bull Exp Biol Med. 2012; 10:435-8. [in Russian]. 